\title{
The applicability of the UCLA loneliness scale in South Africa: Factor structure and dimensionality
}

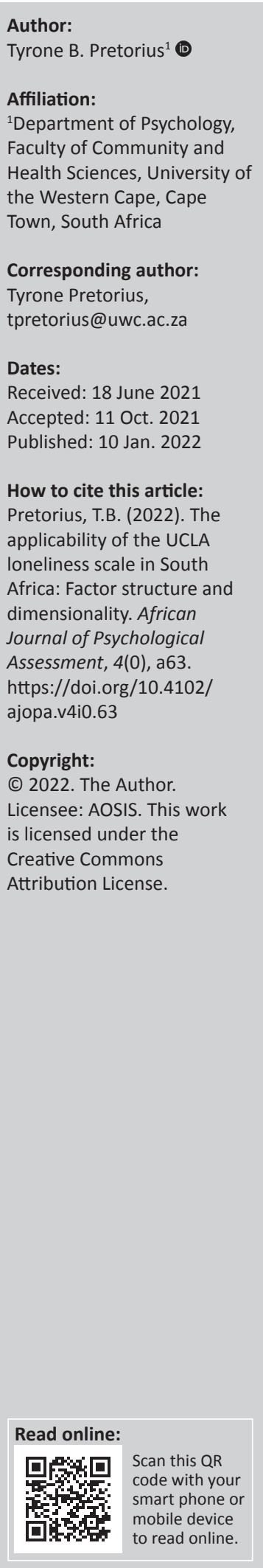

This study examines the generalisability of the University of California Los Angeles Loneliness Scale Version 3 (UCLA-LS3) in a South African sample of young adults. In particular, it examined the normative data, reliability, and factor structure of this scale. The participants were young adults $(N=337)$ who were randomly sampled from a university population and they responded to the UCLA Loneliness Scale. It was found that the sample had higher loneliness scores than those reported in the literature, potentially suggesting that loneliness may be a significant mental health concern amongst this group. Women reported higher levels of loneliness than men. Reliability analysis (Cronbach's alpha) and analysis of the influence of individual items on the mean, variance, and alpha demonstrated that UCLA-LS3 had highly satisfactory internal consistency in the sample. Confirmatory factor analysis (CFA) was used to test four conceptualisations of the factor structure of UCLA-LS3: a one-factor model, a correlated three-factor model, a bifactor model with two subscales, and a bifactor model with three subscales. Notably, CFA demonstrated that the two bifactor models are a better fit than the one-factor and correlated three-factor models and that the bifactor model with three subscales is marginally a better fit than the bifactor model with two subscales. Ancillary bifactor analysis confirmed the dimensionality of the scale as sufficient variance was accounted for by the three subscales, after the variance attributable to the total scale was partitioned out. Therefore, UCLA-LS3 is best conceptualised as comprising of three subscales (isolation, relational connectedness, collective connectedness), in addition to a total scale.

Keywords: bifactor; COVID-19; loneliness; UCLA-LS3; reliability; South Africa.

Recently, the onset of the novel Corona Virus Disease 2019, referred to as COVID-19, and its rapid outbreak led to a global public health crisis (Luchetti et al., 2020). In the absence of a vaccine or cure, the World Health Organization (WHO) recommended social distancing, self-isolation, and quarantine to slow the spread of the virus and in this way reduce the burdens imposed on healthcare systems (WHO, 2020).

Loneliness has been identified as one of the most salient mental health consequences of the COVID-19 pandemic and the prevention measures resulting from it, like stay-at-home directives, limited in-person contact with family and friends, self-isolation and quarantine, etc. (Killgore, Cloonen, Taylor, \& Dailey, 2020; Rosenberg, Luetke, Hensel, Kianersi, \& Herbenick, 2020). Loneliness is defined as the discrepancy between one's actual levels of interaction with others and the desired levels thereof (Perlman \& Peplau, 1981). It is a subjective and distressing emotional state characterised by the perception of social isolation and sense of feeling alone. Even prior to COVID-19, loneliness was a risk factor for adverse physical and mental illnesses, including depression, cardiovascular disease, Alzheimer's disease, and other problems such as suicide, lower life satisfaction, reduced work performance and mortality (Chang et al., 2017; Chiao, Chen, \& Yi, 2019; Maguire, Hanly, \& Maguire, 2019). Hence, the assessment of loneliness has been identified as a significant public health issue (Killgore et al., 2020). According to evolutionary theory, loneliness serves as a signal to reconnect with significant others (Luchetti et al., 2020). However, in the context of a pandemic characterised by self-quarantine and stay-at-home directives, such reconnection may not be possible. This is even more so in developing contexts where access to digital technologies that assists with social connectedness are not affordable or available to a large part of the population (Padfmanabhanunni \& Pretorius, 2021). In such circumstances, feelings of loneliness may become aggravated and evoke further distress (Luchetti et al., 2020).

The most widely used self-report measure for the assessment of loneliness amongst adults and adolescents is the UCLA Loneliness Scale (UCLA-LS: Russell, 1996; Russell, Peplau, \& Ferguson, 1978; Russell, Peplau, \& Cutrona, 1980). The UCLA-LS operationalises loneliness as an undifferentiated unitary state that varies in intensity and arises from perceived deficits in social 
relationships (Russell, 1996). Three versions exist for this scale. The original version (Russell et al., 1978) contained 20 negatively worded items that assessed an individual's perceived experience of loneliness. Because all items were negatively worded, it produced systematic bias in responses. A revised version of the scale was then developed (Russell et al., 1980) that contained 10 negatively worded and 10 positively worded items. However, studies involving exploratory factor analysis (EFA) reported a variety of factor solutions including one-factor (Pretorius, 1993), two-factor with positive and negative items (Mahon et al., 1995), threefactor (Dussault, Fernet, Austin, \& Leroux, 2009) as well as four- and five-factor structures (e.g. Neto, 1992). These inconsistencies limited the utility of the scale as an assessment tool and prompted a revision (Russell, 1996).

The third version of the scale (UCLA-LS3: Russell, 1996) contains 11 negative and 9 positive items. The internal consistency, test-retest reliability and discriminant and convergent validity of the UCLA-LS3 has been supported by several studies (e.g. Auné, Abal, \& Attorresi, 2020; LópezRamos, Navarro-Pardo, Fernández Muñoz, \& Da Silva Pocinho, 2017). Further confirming its utility over the two previous versions, the UCLA-LS3 has been adapted and validated in various cultural contexts and countries (e.g. France, Germany, Greece, Japan, and Russia: Perlman \& Peplau, 1998; Argentina: Auné et al., 2020; Saudi Arabia: AlNajjar \& Dodeen, 2017; Spain: Sancho, Pinazo-Hernandis, Donio-Bellegarde, \& Tomás, 2020; Poland: Kwiatkowska, Rogoza, \& Kwiatkowska, 2018; Iran: Zarei, Memari, Moshayedi, \& Shayestehfar, 2016; Portugal: López-Ramos et al., 2017; Palestine: Nazzal, Cruz, \& Neto, 2018).

With reference to the scale's dimensionality, Russell (1996) maintained that the UCLA-LS3 has a unidimensional structure. However, one-factor (e.g. Lasgaard, 2007), twofactor (e.g. Dodeen, 2015), three-factor (e.g. Hawkley, Browne, \& Cacioppo, 2012; Shevlin, Murphy, \& Murphy, 2014), and bifactor solutions (e.g. Auné et al., 2020) have been reported thus suggesting a possible multidimensional factor structure. This multidimensionality implies that loneliness is a complex experience that involves various types of relationships and interactions. In accounting for these findings, Russell (1996) maintained that the scale measures a unitary state which can be reached via deficits in different relationships and social networks. Nevertheless, the model that has received the most support consists of three factors namely, isolation (i.e. the feelings of isolation that underlie loneliness), social connectedness which refers to the need for meaningful group connectedness and relational connectedness which refers to the need for social contact and close friendships (Hawkley et al., 2012; Shevlin et al., 2014).

Given that loneliness has been identified as a priority mental health problem during the COVID-19 pandemic and the UCLA-LS3 continues to be the most widely used instrument for assessing loneliness, it remains important to understand how the scale performs in different cultural contexts. Responses to psychological scales such as the UCLA-LS3 is influenced by the culture, age and socio-economic circumstances of the respondent (Dodeen, 2014; Durak \& Senol-Durak, 2010). Younger people and those from disadvantaged backgrounds may be more susceptible to wording effects (Wouters et al, 2012). Hence, it remains relevant to understand how the scale performs in different populations.

This study aims to provide insights into the manifestation of loneliness amongst a sample of young adults during COVID-19 and to investigate the psychometric properties of the UCLA-LS in this population group. Measuring instruments such as the UCLA-LS3 that are developed in the fields of personality or social psychology typically have a well-defined exploratory factor analytic structure (Boffo, Mannarini, \& Munari, 2012). However, they are often not adequately supported by Confirmatory Factor Analysis (CFA) and may not reach minimum standards of fit (Boffo et al., 2012). Research on loneliness in developing contexts like South Africa requires suitable instruments with sound psychometric properties that have cross-national applicability. Heppner, Pretorius, Wei, Lee and Wang (2002) argued that such cross-national applicability of instruments would allow building a more comprehensive knowledge base by searching for psychological universals (i.e. etic approach) and identifying culturally specific constructs, which are useful for identifying and explaining cultural differences (i.e. emic approach). Overall, the research findings on factor structure of the UCLA-LS3 have been equivocal. Hence, this study aims to examine the normative data, reliability, and factor structure of the scale in the South African context.

\section{Method \\ Participants}

The study participants were young adults who were doing undergraduate studies at a university in the province of the Western Cape, South Africa. The study design was crosssectional in nature, and the sample of young adults $(N=337)$ were randomly sampled from the university student population. In terms of gender, $77.2 \%$ of the sample were female and $22.0 \%$ were male, whilst $0.8 \%$ self-identified as transgender or binary. The mean age of the sample was 21.95 years $(\mathrm{SD}=4.7)$.

\section{Instruments}

In addition to a demographic questionnaire, all the participants completed the UCLA Loneliness Scale (UCLA-LS3; Russell, 1996).

The UCLA-LS3 consists of 20-items scored on a 4-point scale that ranged from I never feel this way (1) to I often feel this way (4). The UCLA-LS3 is purportedly a measure of an individual's subjective feelings of loneliness and feelings of social isolation. Generally, good internal consistency reliability has been reported for the UCLA-LS3 $(\alpha 0.94$ to 0.96 : Doğan, Çötok, \& Tekin, 2011). The UCLA-LS3 has also been 
previously used in one South African study, which reported a Cronbach's alpha of 0.77 (Pretorius, 1993).

\section{Procedure}

Google forms were used to construct an electronic version of the UCLA-LS3 and the link was distributed to a random sample of students via an email by the Registrar's office. The participants could access and complete the link between March and June 2021.

\section{Data analysis}

In this study, CFA was used to examine four operationalisations of the structure of UCLA-LS3. In CFA, the factors are regarded as latent variables which are represented by the items that are the observed measurements (Bentler, 1995). The four models of the structure of the UCLA-LS3 that were assessed, were a model representing only a total loneliness score (one-factor model), a model representing the structure of the UCLA-LS3 as three correlated subscales (correlated three-factor model), and two models in which the structure of the scale is hypothesised to have both a total scale as well as subscales (bifactor model). The first bifactor model postulates that the UCLA-LS3 consists of a total scale (general factor) with two uncorrelated subscales (specific factors) reflecting the variance amongst clusters of items (Mansolf \& Reise, 2017), whereas the second bifactor model conceptualised UCLALS3 as consisting of a total scale (general factor) with three uncorrelated subscales (specific factors).

In CFA, the chi-square statistic $\left(\chi^{2}\right)$ is used to determine whether the proposed model fits the observed data. However, it has been reported that $\chi^{2}$ test is very sensitive to the violations of distributional assumptions and is affected by sample size (Jöreskog, Olsson, \& Wallentin, 2016), It has, therefore, been recommended (Kline, 2005) that the following fit indices should also be reported: the root-mean-square error of approximation (RMSEA; $\leq 0.08$ indicates good fit), comparative fit index (CFI; $\leq 0.90$ indicates good fit), and standardised root-mean-square residual (SRMR; $\leq 0.08$ indicates good fit). Other indices that are commonly reported include the goodness-of-fit index (GFI; $\geq 0.95$ indicates good fit) and Tucker-Lewis index (TLI; $\geq 0.90$ indicates good fit; Byrne, 1994; Hu \& Bentler, 1999). When models are being compared, it is recommended that indices, which is used to compare models such as the Akaike information criterion (AIC), also be included (Arbuckle, 2012). In general, the model with the lowest AIC value is considered to have the best fit. The CFA analyses were conducted using IBM SPSS Amos (version 26; IBM Corp., Armonk, NY, USA).

It has been pointed out that fit indices do not necessarily address the dimensionality of a scale and may lead to incorrect conclusions about the structure of a scale (Pretorius, 2021). We used the Bifactor Indices Calculator (Dueber, 2017) to calculate additional bifactor measures to address the dimensionality of the UCLA-LS3. These measures include (1) explained common variance (ECV), which refers to the proportion of variance accounted for by the specific factor; (2) McDonald's omega, which is regarded as an alternative to coefficient alpha, as an estimate of reliability (omegaS for subscales); and (3) omega hierarchical (omegaH), which reflects the percentage of variance in total scores that is the result of individual differences on the general factor. An omegaH $>0.80$ reflects, that irrespective of good fit indices, the scale is largely unidimensional. With respect to specific factors (subscales), omegaHS reflects the percentage of systematic variance of the specific factors, after the variance accounted for by the general factor is excluded (Rodriguez, Reise, \& Haviland, 2016).

\section{Ethical considerations}

Ethical approval for this study was obtained from the Humanities and Social Sciences Research Committee of the University of the Western Cape (ethics reference number: HS20/5/1). The participants completed the survey anonymously. The first item in the electronic survey gave participants the opportunity to provide informed consent. At the end of the survey the contact details for the South African Depression and Anxiety Group and the Centre for Student Counselling and participants were urged to reach out to those services if they experienced any distress during completion of the questionnaire.

\section{Results \\ Descriptive statistics}

The mean loneliness score $(M=49.1 ; S D=11.6)$ was much higher than that previously found in a similar sample from South Africa (Pretorius, 1993; $M=38.8, S D=7.8$ ), as well as those in the literature, $M=34$ to 38: e.g. Auné et al., 2020; Hartshorne, 1993; Shevlin et al., 2015). The score was also higher than that found in a COVID-19 study (Killgore et al., 2020; $M=43.8, S D=13.5$ ).

\section{Reliability}

The reliability of the UCLA-LS3 (alpha $=0.923$, omega $=$ 0.924) was satisfactory, and compared favourably with the reliabilities documented in previous studies (e.g. Tull et al., 2020). Table 1 shows the impact of each item, if it were to be deleted, on the alpha coefficient, variance and mean, as well as the item-total correlation.

The correlations between individual items and the total score ranged between 0.26 and 0.73 , whereas the item-deleted alphas were all 0.92, except for Item 17, which had an alpha of 0.93 . Except for one related to items 8 and 15, the interitem correlations ranged between 0.11 and 0.87 and were all significant. The mean interitem coefficient was 0.37 . Overall, the evidence for internal consistency was highly satisfactory.

\section{Factor structure}

In this study, we compared a one-factor model (UCLA-LS3 total score), a correlated three-factor model, a bifactor model 
TABLE 1: The influence of items on the mean, variance, and alpha and item-total correlation.

\begin{tabular}{lcccc}
\hline Item & $\begin{array}{c}\text { Scale mean if } \\
\text { item deleted }\end{array}$ & $\begin{array}{c}\text { Scale variance if item } \\
\text { deleted }\end{array}$ & $\begin{array}{c}\text { Item-total } \\
\text { correlation }\end{array}$ & $\begin{array}{c}\text { Alpha if item } \\
\text { deleted }\end{array}$ \\
\hline UCLA1 & 46.87 & 124.48 & 0.50 & 0.92 \\
UCLA2 & 46.42 & 122.52 & 0.58 & 0.92 \\
UCLA3 & 46.66 & 118.40 & 0.73 & 0.92 \\
UCLA4 & 46.44 & 119.67 & 0.66 & 0.92 \\
UCLA5 & 46.91 & 123.35 & 0.50 & 0.92 \\
UCLA6 & 46.83 & 122.86 & 0.59 & 0.92 \\
UCLA7 & 46.50 & 119.67 & 0.67 & 0.92 \\
UCLA8 & 46.34 & 123.21 & 0.59 & 0.92 \\
UCLA9 & 47.20 & 126.36 & 0.45 & 0.92 \\
UCLA10 & 47.01 & 122.16 & 0.68 & 0.92 \\
\hline UCLA11 & 46.36 & 121.25 & 0.65 & 0.92 \\
\hline UCLA12 & 46.59 & 121.30 & 0.61 & 0.92 \\
UCLA13 & 46.24 & 119.95 & 0.65 & 0.92 \\
\hline UCLA14 & 46.36 & 120.66 & 0.68 & 0.92 \\
UCLA15 & 46.91 & 126.22 & 0.39 & 0.92 \\
\hline UCLA16 & 46.88 & 121.69 & 0.61 & 0.92 \\
\hline UCLA17 & 46.12 & 129.34 & 0.26 & 0.93 \\
UCLA18 & 46.26 & 122.17 & 0.62 & 0.92 \\
UCLA19 & 47.01 & 119.69 & 0.67 & 0.92 \\
\hline UCLA20 & 47.07 & 120.51 & 0.65 & 0.92 \\
\hline
\end{tabular}

with two subscales, and a bifactor model with three subscales. Notably, the two or three subscales conceptualisations of the bifactor models were based on previous factor analysis studies (Hawkley et al., 2012; Shevlin et al., 2014; Wilson, Cutts, Lees, Mapungwana, \& Maunganidze, 1992), which identified a two-factor solution (Social Others = SO; Intimate Others $=\mathrm{IO}$ ) as well as a threefactor solution (Collective Connectedness $=\mathrm{CC}$; Isolation $=$ I; Relational Connectedness $=\mathrm{RC}$ ). Table 2 shows the item groupings and factor labelling for the one-factor model, the three-factor model, and the two bifactor models based on these studies.

The four representations of the factor structure of UCLA-LS3 and the results of the CFA are shown in Figures 1-4.

In the one-factor model, it is assumed that a single factor (total loneliness score) best explains the variance amongst the items, whereas the correlated three-factor model presumes that three related factors account for the variance. The bifactor model, in contrast, presumes that a general factor (loneliness) explains a certain proportion of the variance, whereas two/three specific factors (subscales) account for the remaining variance. Table 3 reflects the fit indices for the three models.

As detailed in Table 3, the one-factor model failed to meet any of the criteria indicating a good fit. The correlated threefactor model had much better indices than those of the onefactor model, but they were marginally lower than those of the bifactor models, with only marginal differences in the fit indices of the two bifactor models. In both the two-subscale and the three-subscale bifactor models, TLI, CFI, and RMSEA were identical (TLI $=0.92, \mathrm{CFI}=0.94$, and RMSEA $=0.07$, indicating a reasonable fit. However, in terms of GFI and SRMR, the three-subscale bifactor model showed a better fit.
TABLE 2: Item groupings for the one-factor and two bifactor models.

\begin{tabular}{lccc}
\hline Item & One-factor & $\begin{array}{c}\text { Bifactor: two } \\
\text { subscales }\end{array}$ & $\begin{array}{c}\text { Bifactor: three } \\
\text { subscales }\end{array}$ \\
\hline 1. In tune with others & $\mathrm{L}$ & $\mathrm{SO}$ & $\mathrm{CC}$ \\
\hline 2. Lack companionship & $\mathrm{L}$ & $\mathrm{IO}$ & $\mathrm{I}$ \\
3. No one to turn to & $\mathrm{L}$ & $\mathrm{IO}$ & $\mathrm{I}$ \\
4. Alone & $\mathrm{L}$ & $\mathrm{IO}$ & $\mathrm{I}$ \\
5. Part of a group & $\mathrm{L}$ & $\mathrm{SO}$ & $\mathrm{CC}$ \\
6. A lot in common with people & $\mathrm{L}$ & $\mathrm{SO}$ & $\mathrm{CC}$ \\
7. Not close to anyone & $\mathrm{L}$ & $\mathrm{IO}$ & $\mathrm{I}$ \\
8. Interests not shared & $\mathrm{L}$ & $\mathrm{IO}$ & $\mathrm{I}$ \\
9. An outgoing person & $\mathrm{L}$ & $\mathrm{SO}$ & $\mathrm{CC}$ \\
10. People you feel close to & $\mathrm{L}$ & $\mathrm{SO}$ & $\mathrm{RC}$ \\
11. Left out & $\mathrm{L}$ & $\mathrm{IO}$ & $\mathrm{I}$ \\
12. Relationships not meaningful & $\mathrm{L}$ & $\mathrm{IO}$ & $\mathrm{I}$ \\
13. No one really knows you & $\mathrm{L}$ & $\mathrm{IO}$ & $\mathrm{I}$ \\
14. Isolated from others & $\mathrm{L}$ & $\mathrm{IO}$ & $\mathrm{I}$ \\
15. Can find companionship & $\mathrm{L}$ & $\mathrm{SO}$ & $\mathrm{RC}$ \\
16. People who understand you & $\mathrm{L}$ & $\mathrm{IO}$ & $\mathrm{RC}$ \\
17. Feel shy & $\mathrm{L}$ & $\mathrm{IO}$ & $\mathrm{I}$ \\
18. People are not with you & $\mathrm{L}$ & $\mathrm{IO}$ & $\mathrm{I}$ \\
19. People I can talk to & $\mathrm{L}$ & $\mathrm{SO}$ & $\mathrm{RC}$ \\
20. People I can turn to & $\mathrm{L}$ & $\mathrm{SO}$ & $\mathrm{RC}$ \\
\hline
\end{tabular}

Note: L, Loneliness; SO, Social Others; IO, Intimate Others; CC, Collective Connectedness; I, Isolation; RC, Relational Connectedness.

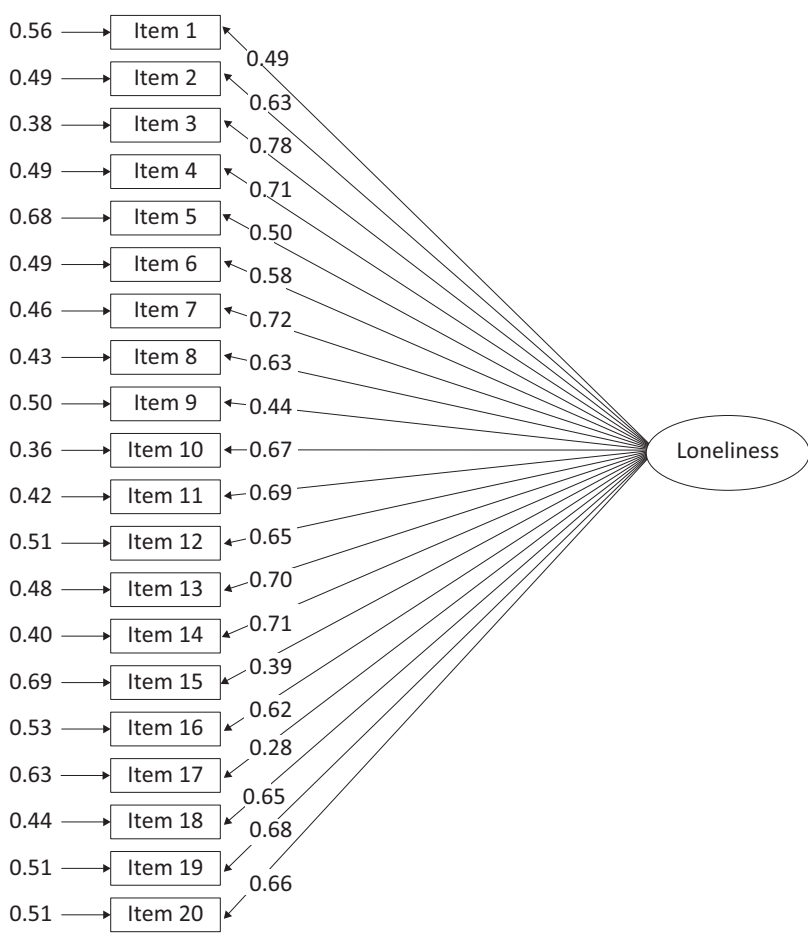

Note: The rectangles are measured variables, whereas the ellipse is a latent construct. FIGURE 1: One-factor model of UCLA-LS3.

In addition, the model comparison index (i.e. AIC) was lower for the three-subscale bifactor model than for the twosubscale bifactor model (487.13 in comparison to 506.37), indicating a slightly better fit.

Despite the evidence provided by the CFA in relation to the superiority of the bifactor structure for UCLA-LS3, the CFA did not address the dimensionality of the scale. More specifically, the CFA did not clarify the relative proportion of 


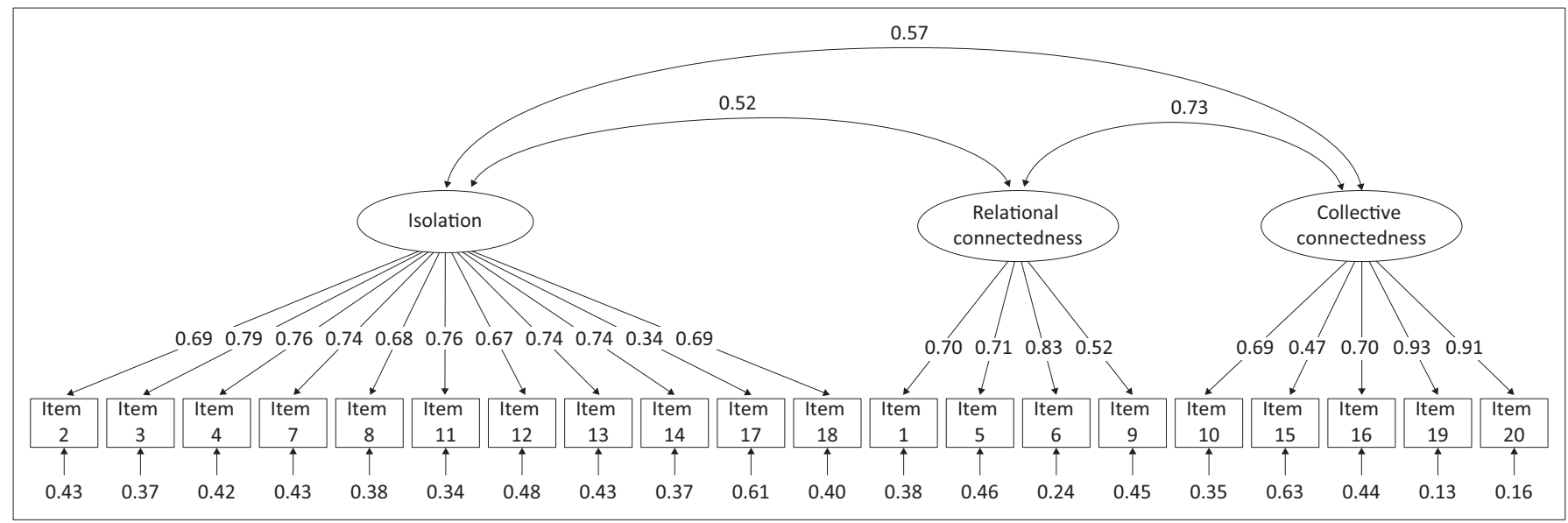

Note: The rectangles are measured variables, whereas the ellipses are latent constructs.

FIGURE 2: Correlated three-factor model.

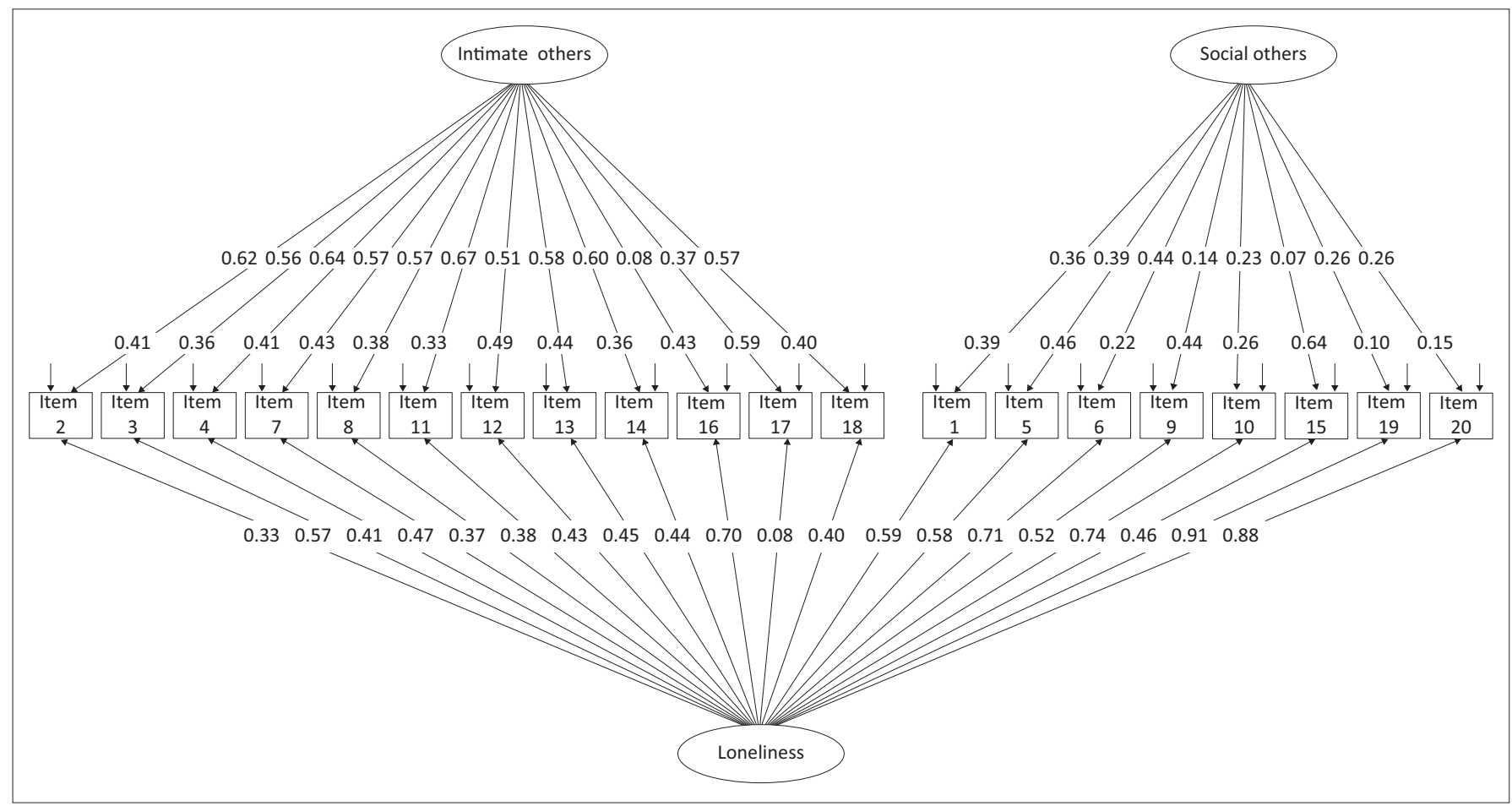

Note: The rectangles are measured variables, whereas the ellipses are latent constructs.

FIGURE 3: Bifactor model of UCLA-LS3: Two subscales.

variance accounted for by the total scale and subscales. In this regard, Rodriguez et al. (2016), for example, urged researchers to use bifactor indices, over and above fit indices to examine the dimensionality of instruments. Table 4 reflects the bifactor indices for the UCLA-LS3.

The ECV is the percentage of all common variance for all items explained by a factor. Table 4 indicates that the general factor (loneliness) explained 59\% and 57\%, respectively, of the common variance in the two-subscale and three-subscale bifactor models. Therefore, specific factors (IO and SO in the case of the two-subscale bifactor model and I, RC, and CC in the case of the three-subscale bifactor model) explained $41 \%$ and $43 \%$ of the variance, respectively. This result confirms the multidimensionality of UCLA-LS3, as the specific factors accounted for sufficient variance, after the variance accounted for by the general factor was taken into consideration. In addition, omegaH, which reflects the percentage of variance in total scores accounted for by the general factor, was below the cut-off point suggested in the literature (omegaH $=0.68$ and 0.69). Reise, Bonifay and Haviland (2013) proposed that when omegaH is greater than 0.80 , the scale can be considered essentially unidimensional.

Lastly, the omegaS coefficient, which is a model-based estimate of reliability, further confirmed that the various subscales in the two-subscale and three-subscale bifactor models $(\mathrm{IO}$ : omegaS $=0.92$; SO: omegaS $=0.90$; I: omegaS $=0.90 ; \mathrm{RC}$ : omegaS $=0.89 ; \mathrm{CC}=0.79)$ demonstrated sufficient reliability. 


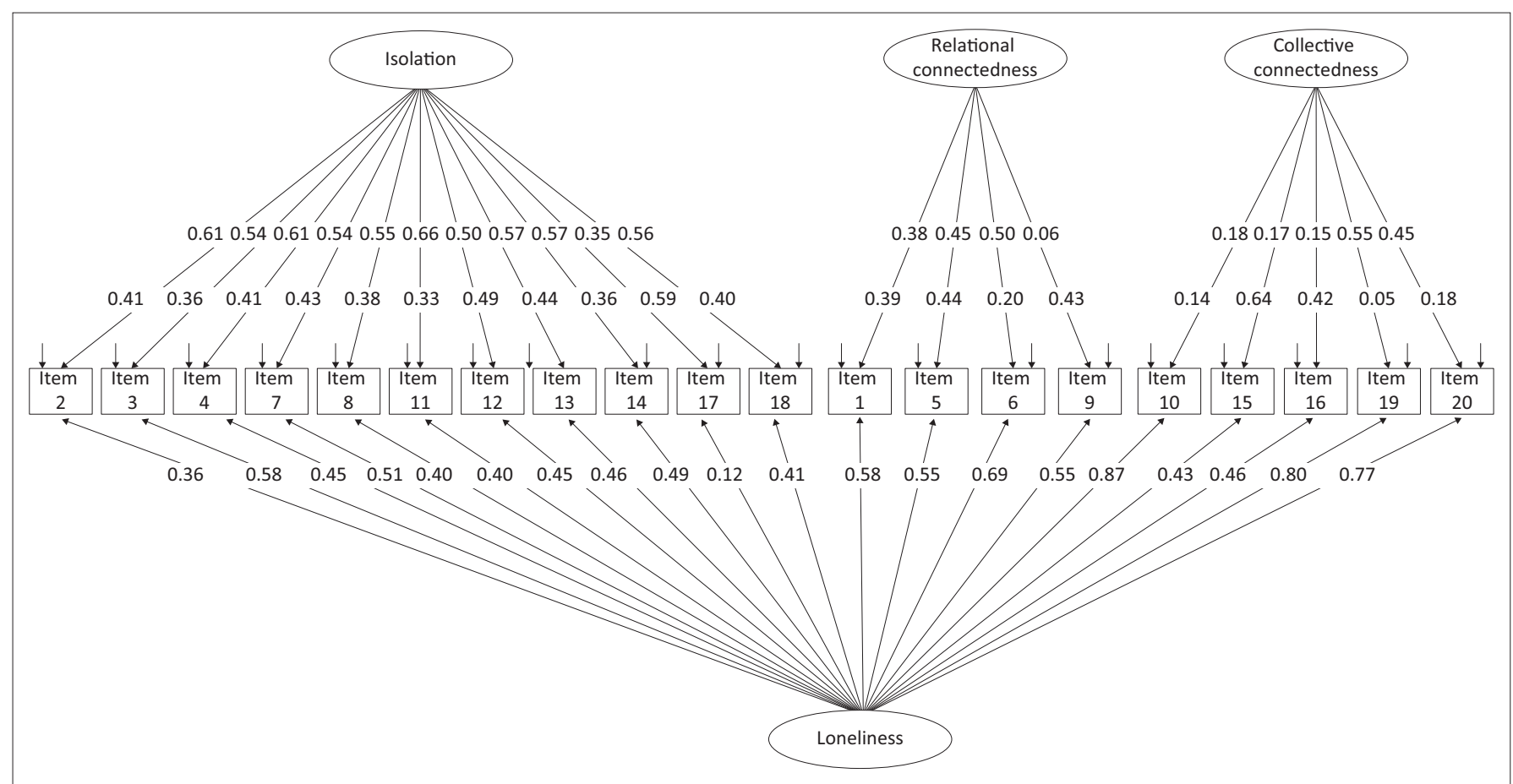

Note: The rectangles are measured variables, whereas the ellipses are latent constructs.

FIGURE 4: Bifactor model of UCLA-LS3: Three subscales.

TABLE 3: Fit indices for three models of the structure of UCLA-LS3.

\begin{tabular}{lccccc}
$\begin{array}{l}\text { Goodness- } \\
\text { of-fit } \\
\text { indices }\end{array}$ & $\begin{array}{c}\text { Best fit } \\
\text { indicator }\end{array}$ & $\begin{array}{c}\text { One-factor } \\
\text { model }\end{array}$ & $\begin{array}{c}\text { Correlated } \\
\text { three-factor } \\
\text { model }\end{array}$ & $\begin{array}{c}\text { Bifactor } \\
\text { model: two } \\
\text { subscales }\end{array}$ & $\begin{array}{c}\text { Bifactor } \\
\text { model: three } \\
\text { subscales }\end{array}$ \\
\hline$\chi^{2}(d f)$ & $1,309.70(170)$ & $524.76(167)$ & $386.37(150)$ & 367.13 \\
$p$-value & Nonsignificant & $p<0.001$ & $p<0.001$ & $p<0.001$ & $p<0.001$ \\
GFI & $>0.95$ & 0.62 & 0.86 & 0.89 & 0.90 \\
TLI & $>0.90$ & 0.65 & 0.89 & 0.92 & 0.92 \\
CFI & $>0.90$ & 0.69 & 0.90 & 0.94 & 0.94 \\
RMSEA & $<0.08$ & 0.14 & 0.08 & 0.07 & 0.07 \\
SRMR & $<0.08$ & 0.09 & 0.05 & 0.04 & 0.03 \\
AIC & Lower levels & $1,389.70$ & 610.76 & 506.37 & 487.13 \\
\hline
\end{tabular}

Note: $\chi^{2}=$ chi-square statistic; GFI, goodness-of-fit index; TLI, Tucker-Lewis index; CFI, comparative fit index; RMSEA, root-mean-square error of approximation; SRMR, standardized root-mean-square residual; AIC, Akaike information criterion.

Both the CFA and bifactor indices provide support for the use of UCLA-LS3, as consisting of a total scale as well as either two or three subscales, although the three-subscale bifactor model fit the data marginally better than the two-subscale bifactor model.

\section{Discussion}

The aim of the present study was to extend current research on UCLA-LS3 by investigating its applicability to a sample of young adults in South Africa. The current study, hence, focused on the psychometric properties and normative data of the UCLA-LS3.

There were several important findings. Firstly, we found that the participants in the current study reported higher levels of loneliness than those found in prior studies (e.g. Killgore et al., 2020), thus suggesting a potential mentalhealth crisis. Such unprecedented levels of loneliness can be attributed to COVID-19 preventive measures, such as
TABLE 4: Dimensionality indices for UCLA-LS3.

\begin{tabular}{lccc}
\hline Bifactor model & $\mathrm{ECV}_{\dagger}^{\dagger}$ & Omega/omegaS & $\begin{array}{c}\text { OmegaH/ } \\
\text { omegaHS } \S\end{array}$ \\
\hline Two-subscale bifactor model & & & \\
Loneliness & 0.59 & 0.94 & 0.68 \\
IO & 0.35 & 0.92 & 0.56 \\
SO & 0.07 & 0.90 & 0.03 \\
Three-subscales bifactor & & & \\
model & & & 0.69 \\
Loneliness & 0.57 & 0.94 & 0.58 \\
I & 0.32 & 0.92 & 0.08 \\
RC & 0.06 & 0.89 & 0.20 \\
CC & 0.06 & 0.79 & \\
\hline
\end{tabular}

Note: $\dagger, E C V$, explained common variance; $\downarrow$, Omega for total scale and omegaS for subscales; $\S$, OmegaH for total scale and omegaHS for subscales; IO, Intimate Others; SO, Social Others; I, Isolation; RC, Relational Connectedness; CC, Collective Connectedness.

prolonged prohibition of in-person social contact and social distancing directives. They could also be the result of disparities in the access to digital technologies that can be used to circumvent these restrictions and maintain social connectedness. Women reported higher loneliness mean scores then men, a finding that confirms previous research on gender differences in loneliness (e.g. Wang \& Zhao 2020). Such higher levels of loneliness amongst women may be because of the impact of gender role socialisation, which contributes to women prioritising affiliations with friends and family. Disruptions in the access to these networks as a result of stay-at-home directives may also contribute to increased psychological distress for women (Li \& Wang, 2020).

Secondly, reliability analysis (Cronbach's alpha) and analysis of the influence of individual items on the mean, variance, and alpha demonstrated that UCLA-LS3 exhibited highly satisfactory internal consistency in the sample. The obtained 
alpha coefficient was comparable to those reported in the literature (e.g. Ausín, Muñoz, Martín, Pérez-Santos, \& Castellanos, 2019; Pikea, Parpa, Tsilika, Galanos, \& Mystakidou., 2016). The results, therefore, support the use of UCLA-LS3 in culturally diverse contexts, and the findings suggest that meaningful comparisons can be drawn across countries.

Thirdly, the CFA demonstrated that the two bifactor models were a better fit than the one-factor model. The bifactor indices also demonstrated that the two and three subscales of the bifactor models account for sufficient variance after the variance accounted for by the general factor was considered. The bifactor model with three subscales had marginally better fit indices than those of the bifactor model with two specific subscales. These results, therefore, support the findings of Hawkley et al. (2012) and Shevlin et al. (2014). Thus, it can be concluded that UCLA-LS3 is best conceptualised as comprising of three subscales (isolation, relational connectedness, collective connectiveness), in addition to a total scale. The study confirms that loneliness, as measured by the UCLA-LS3, is a multidimensional concept. It also provides support for the future use of the scale in the South African context, especially related to the assessment of loneliness during the COVID-19 pandemic.

\section{Limitations}

This study has some limitations. The first limitation is the small sample size. However, the sample size is still within the rule of thumb of 10 cases per variable (Wolf, Harrington, Clark, \& Miller, 2013). Moreover, the unequal gender sample, whilst consistent with the demographics in the university, prevented gender comparisons of models.

\section{Conclusion}

This study provides support for the generalisability of UCLA-LS3 in a South African sample of young adults and paves the way for its further use in South African samples. The scale demonstrated sound reliability, and the bifactor analysis confirmed the multidimensionality thereof.

\section{Acknowledgements Competing interests}

The author declares that he has no financial or personal relationships that may have inappropriately influenced him in writing this research article.

\section{Author's contributions}

T.B.P. is the sole author of this article.

\section{Funding information}

This research received no specific grant from any funding agency in the public, commercial or not-for-profit sectors.

\section{Data availability}

The data will be available at https:/ /uwc.figshare.com/.

\section{Disclaimer}

The views and opinions expressed in this article are those of the author and do not necessarily reflect the official policy or position of any affiliated agency of the author.

\section{References}

AlNajjar, A., \& Dodeen, H.A.M.Z.E.H. (2017). Factor structure of the Arabic version of the UCLA loneliness scale. International Journal of Research in Humanities, Arts and Literature, 5(9), 171-184. https://doi.org/10.1080/03601277.2015.10 65688

Arbuckle, J.L. (2012), Amos 21.0 User's Guide. Mount Pleasant: Amos Development Corporation.

Auné, S.E., Abal, F.J.P., \& Attorresi, H.F. (2020). Modeling of the UCLA Loneliness Scale according to the multidimensional item response theory. Current Psychology, 2, 1-8. https://doi.org/10.1007/s12144-020-00646-y

Ausín, B., Muñoz, M., Martín, T., Pérez-Santos, E., \& Castellanos, M.Á. (2019). Confirmatory factor analysis of the Revised UCLA Loneliness Scale (UCLA LS-R) in individuals over 65. Aging \& Mental Health, 23(3), 345-351. https://doi.org/10.1 080/13607863.2017.1423036

Bentler, P.M. (1995). EQS: Structural equations program manual. Multivariate Software. Los Angeles: University of California.

Boffo, M., Mannarini, S., \& Munari, C. (2012). Exploratory structure equation modeling of the UCLA loneliness scale: a contribution to the Italian adaptation. TPM: Testing, Psychometrics, Methodology in Applied Psychology, 19(4), 345-363. https://doi.org/10.4473/TPM19.4.7

Byrne, B.M. (1994). Testing for the factorial validity, replication, and invariance of a measuring instrument: A paradigmatic application based on the Maslach Burnout Inventory. Multivariate Behavioral Research, 29, 289-311. https://doi. org/10.1207/s15327906mbr2903_5

Chang, E.C., Wan, L., Li, P., Guo, Y., He, J., Gu, Y., ... Batterbee, C.N.H. (2017). Loneliness and suicidal risk in young adults: Does believing in a changeable future help and suicidal risk in young adults: Does believing in a changeable future help
minimize suicidal risk amongst the lonely? The Journal of Psychology, 151(5), minimize suicidal risk amongst the lonely? The Journal of
453-463. https://doi.org/10.1080/00223980.2017.1314928

Chiao, C., Chen, Y.H., \& Yi, C.C. (2019). Loneliness in young adulthood: Its intersecting forms and its association with psychological well-being and family characteristics in Northern Taiwan. PLoS One, 14(5), e0217777. https://doi.org/10.1371/journal. pone.0217777

Dodeen, H. (2015). The effects of positively and negatively worded items on the factor structure of the UCLA loneliness scale. Journal of Psychoeducational Assessment, 33(3), 259-267. https://doi.org/10.1177\%2F0734282914548325

Doğan, T., Çötok, N.A., Tekin, E.G. (2011). Reliability and validity of the Turkish Version of the UCLA Loneliness Scale (ULS-8) amongst university students. Procedia-Social and Behavioral Sciences, 15, 2058-2062. https://doi.org/10.1016/j.sbspro. 2011.04.053

Dueber, D.M. (2017). Bifactor indices calculator: A Microsoft excel-based tool to calculate various indices relevant to bifactor CFA models. Retrieved from http:// sites.education.uky.edu/apslab/resources/

Durak, M., \& Senol-Durak, E. (2010). Psychometric qualities of the UCLA loneliness scale-version 3 as applied in a Turkish culture. Educational Gerontology 36(10-11), 988-1007. https://doi.org/10.1080/03601271003756628

Dussault, M., Fernet, C., Austin, S. \& Leroux, M. (2009). Revisiting the factorial validity of the Revised UCLA Loneliness Scale: A test of competing models in a sample of teachers. Psychological Reports, 105(3), 849-856. https://doi.org/ 10.2466\%2FPR0.105.3.849-856

Hartshorne, T.S. (1993). Psychometric properties and confirmatory factor analysis of the UCLA Loneliness Scale. Journal of Personality Assessment, 61(1), 182-195. https://doi.org/10.1207/s15327752jpa6101_14

Hawkley, L.C., Browne, M.W., \& Cacioppo, J.T. (2005). How can I connect with thee? Let me count the ways. Psychological Science, 16(10), 798-804. https://doi. org/10.1111/j.1467-9280.2005.01617.x

Heppner, P.P., Pretorius, T.B., Wei, M., Lee, D.G., \& Wang, Y.W. (2002). Examining the generalizability of problem-solving appraisal in Black South Africans. Journal of Counseling Psychology, 49(4), 484. https://doi.org/10.1037/00220167.49.4.484

Hu, L.T., \& Bentler, P.M. (1999). Cutoff criteria for fit indexes in covariance structure analysis: Conventional criteria versus new alternatives. Structural Equation Modeling: A Multidisciplinary Journal, 6, 1-55. https://doi.org/10.1080/10705519909540118

Jöreskog K.G., Olsson U.H., Wallentin F.Y. (2016) Confirmatory Factor Analysis (CFA). In: Multivariate Analysis with LISREL. Springer Series in Statistics. Springer, Cham. https://doi.org/10.1007/978-3-319-33153-9_7

Killgore, W.D., Cloonen, S.A., Taylor, E.C., \& Dailey, N.S. (2020). Loneliness: A signature mental health concern in the era of COVID-19. Psychiatry Research, 290, 113-117. https://doi.org/10.1016/j.psychres.2020.113117

Kline, R. B. (2005). Principles and practice of structural equation modeling (2nd ed.). Guilford: New York, NY. 
Kwiatkowska, M.M., Rogoza, R., \& Kwiatkowska, K. (2018, July 25). Analysis of the psychometric properties of the Revised UCLA Loneliness Scale in the Polish adolescent sample. Current Issues in Personality Psychology, 6(2), 164-170. adolescent sample. Current Issues in ht//doi.org/10.5114/cipp.2017.69681

Lasgaard, M. (2007). Reliability and validity of the Danish version of the UCLA Loneliness Scale. Personality and Individual Differences, 42(7), 1359-1366. https://doi.org/10.1016/j.psychres.2020.113117

Li, L.Z., \& Wang, S. (2020). Prevalence and predictors of general psychiatric disorders and loneliness during COVID-19 in the United Kingdom. Psychiatry Research, 291, 113267. https://doi.org/10.1016/j.psychres.2020.113267

López-Ramos, Y., Navarro-Pardo, E., Fernández Muñoz, J.J., \& Da Silva Pocinho, R.F. (2017). Psychometric properties and factor structure of the satisfaction with life scale in an elderly Portuguese students sample. Anales De Psicología/ Annals of Psychology, 34(1), 146-152. https://doi.org/10.6018/analesps.34. 1.267381

Luchetti, M., Lee, J.H., Aschwanden, D., Sesker, A., Strickhouser, J.E., Terracciano, A., \& Sutin, A.R. (2020). The trajectory of loneliness in response to COVID-19. American Psychologist, 75(7), 897-908. https://doi.org/10.1037/amp0000690

Maguire, R., Hanly, P., \& Maguire, P. (2019). Living well with chronic illness: How social support, loneliness and psychological appraisals relate to well-being in a population-based European sample. Journal of Health Psychology, 26(10) 1494-1507. https://doi.org/10.1177/1359105319883923

Mahon, N. E., Yarcheski, T. J., \& Yarcheski, A. (1995). Validation of the revised UCLA Loneliness Scale for adolescents. Research in Nursing \& Health, 18(3), 263-270. https://doi.org/10.1002/nur.4770180309

Mansolf, M., \& Reise, S.P. (2017). When and why the second-order and bifactor models are distinguishable. Intelligence, 61, 120-129. https://doi.org/10.1016/j. intell.2017.01.012

Nazzal, F.I., Cruz, O., \& Neto, F. (2018). Psychometric analysis of the short-form UCLA Loneliness Scale (ULS-6) amongst Palestinian university students. Retrieved from https://www.psycharchives.org/bitstream/20.500.12034/1766/1/ijpr.v11i2.269.pdf

Neto, F. (1992). Loneliness among Portuguese adolescents. Social Behavior and Personality: an International Journal, 20(1), 15-21. https://doi.org/10.2224/ sbp.1992.20.1.15

Padmanabhanunni, A., \& Pretorius, T. B. (2021). The unbearable loneliness of COVID-19: COVID-19-related correlates of loneliness in South Africa in young adults. Psychiatry Research, 296, 113658. https://doi.org/10.1016/j.psychres. 2020.113658

Perlman, D., \& Peplau, L.A. (1981). Toward a social psychology of loneliness. Personal Relationships, 3, 31-56. Retrieved from http://www.academia.edu/download/ 40307428/Perlman__Peplau_81.pdf

Perlman, D., \& Peplau, L. A. (1998). Loneliness. In H. S. Friedman (Ed.) Encyclopedia of Mental Health, Vol 2 (pp. 571-581). Academic Press: Santiago, CA.

Pikea, P., Parpa, E., Tsilika, E., Galanos, A., \& Mystakidou, K. (2016). Psychometric properties of the Greek-University of California, Los Angeles Loneliness Scale-Version 3 in a sample of people with human immunodeficiency virus. World Journal of AIDS, 6(4), 157-168. https://doi.org/10.4236/wja.2016.64018

Pretorius, T.B., (1993). The metric equivalence of the UCLA Loneliness Scale for a sample of South African students. Educational and Psychological Measurement, 53(1), 233-239. https://doi.org/10.1177/0013164493053001026

Pretorius, T.B. (2021). Over reliance on model fit indices in confirmatory factor analyses may lead to incorrect inferences about bifactor models: A cautionary note. African Journal of Psychological Assessment, 3, 4. https://doi.org/10.4102/ ajopa.v3i0.35
Reise, S.P., Bonifay, W.E., \& Haviland, M.G. (2013). Scoring and modeling psychological measures in the presence of multidimensionality. Journal of personality Assessment, 95(2), 129-140. https://doi.org/10.1080/00223891.20 Personality As

Rodriguez, A., Reise, S.P., \& Haviland, M.G. (2016). Evaluating bifactor models: Calculating and interpreting statistical indices. Psychological Methods, 21(2), 137. https://doi.org/10.1037/met0000045

Rosenberg, M., Luetke, M., Hensel, D., Kianersi, S., Herbenick, D. (2020). Depression and loneliness during COVID-19 restrictions in the United States, and their associations with frequency of social and sexual connections. MedRxiv. https:// associations with frequency of social and
doi.org/10.1101/2020.05.18.20101840

Russell, D., Peplau, L.A., \& Cutrona, C.E., (1980). The revised UCLA Loneliness Scale: Concurrent and discriminant validity evidence. Journal of Personality and Social Psychology, 39(3), 472-480. https://doi.org/10.1037/00223514.39.3.472

Russell, D., Peplau, L.A., \& Ferguson, M.L. (1978). Developing a measure of loneliness. Journal of Personality Assessment, 42(3), 290-294. https://doi.org/10.1207/ s15327752jpa4203_11

Russell, D.W. (1996). UCLA Loneliness Scale (Version 3): Reliability, validity, and factor structure. Journal of Personality Assessment, 66(1), 20-40. https://doi. org/10.1207/s15327752jpa6601_2

Sancho, P., Pinazo-Hernandis, S., Donio-Bellegarde, M. \& Tomás, J.M. (2020). Validation of the University of California, Los Angeles Loneliness Scale (version 3) in Spanish older population: An application of exploratory structural equation modeling. Australian Psychologist, 55(3), 283-292. https://doi.org/10.1111/ap.12428

Shevlin, M., Murphy, S., \& Murphy, J. (2014). The latent structure of loneliness testing competing factor models of the UCLA Loneliness Scale in a large adolescent sample. Assessment, 22(2), 208-215. https://doi.org/10. $1177 / 1073191114542596$

Tull, M.T., Edmonds, K.A., Scamaldo, K., Richmond, J.R., Rose, J.P., \& Gratz, K.L., 2020 Psychological outcomes associated with stay-at-home orders and the perceived impact of COVID-19 on daily life. Psychiatry Research, 289, 113098. http://doi. org/10.1016/j.psychres.2020.113098

Wang, C., \& Zhao, H. (2020). The impact of COVID-19 on anxiety in Chinese University students. Frontiers in Psychology, 11, 1168. https://doi.org/10.3389/ fpsyg.2020.01168

Wilson, D., Cutts, J., Lees, I., Mapungwana, S., \& Maunganidze, L. (1992). Psychometric properties of the revised UCLA Loneliness Scale and two short-form measures of loneliness in Zimbabwe. Journal of Personality Assessment, 59, 72-81. https:// doi.org/10.1207/s15327752jpa5901_7

World Health Organisation. (2020). Coronavirus disease (COVID-2019) situation reports. Retrieved from https://www.who.int/emergencies/diseases/novelcoronavirus-2019/ situation-reports

Wolf, E.J., Harrington, K.M., Clark, S.L., \& Miller, M.W. (2013). Sample size requirements for structural equation models: An evaluation of power, bias, and solution propriety. Educational and Psychological Measurement, 73(6), 913-934. https:// doi.org/10.1177\%2F0013164413495237

Wouters, E., Booysen, F. L. R., Ponnet, K., \& Baron Van Loon, F. (2012). Wording effects and the factor structure of the Hospital Anxiety \& Depression Scale in HIV/AIDS patients on antiretroviral treatment in South Africa. PloS one, 7(4), e34881. https://doi.org/10.1371/journal.pone.0034881

Zarei, S., Memari, A.H., Moshayedi, P., \& Shayestehfar, M. (2016). Validity and reliability of the UCLA loneliness scale version 3 in Farsi. Educational Gerontology 42(1), 49-57. https://doi.org/10.1080/03601277.2015.1065688 